\title{
Identification of UV-B-induced microRNAs in wheat
}

\author{
B. Wang*, Y.F. Sun*, N. Song, X.J. Wang, H. Feng, L.L. Huang and \\ Z.S. Kang \\ College of Plant Protection and State Key Laboratory of Crop Stress Biology \\ on Drought Regions, Northwest A\&F University, Yangling, Shaanxi, China \\ *These authors contributed equally to this study. \\ Corresponding author: Z.S. Kang \\ E-mail: kangzs@nwsuaf.edu.cn
}

Genet. Mol. Res. 12 (4): 4213-4221 (2013)

Received October 15, 2012

Accepted May 10, 2013

Published October 7, 2013

DOI http://dx.doi.org/10.4238/2013.October.7.7

\begin{abstract}
MicroRNAs (miRNAs) play critical roles in posttranscriptional gene regulation and act as important endogenous regulators to various stressors. Ultraviolet-B (UV-B) radiation is a major factor influencing crop growth and development. In this study, we isolated a novel wheat miRNA, named Tae-miR6000, and confirmed its expression diversity after UV-B treatments. Additionally, using the Northern blotting technique, we found that six miRNAs were highly responsive to UV-B stress in wheat. Of these six miRNAs, miR159, miR167a, and miR171 were significantly upregulated, and the remaining three miRNAs were downregulated, at different time points after UV-B treatment. This result indicates that miRNAs may be involved in the regulation of targets after induction by UV-B stress. Furthermore, promoter analysis of the UV-B-responsive miRNA genes revealed some light-relevant cis-elements, such as the I-box and G-box. Taken together, the results of this study suggest that wheat miRNAs play important roles in the response to UV-B stress.
\end{abstract}

Key words: Wheat; MicroRNA; Northern blot; UV-B radiation 


\section{INTRODUCTION}

Changing environmental factors can lead to stress and therefore constrain plant productivity (Agarwal et al., 2006). Among these environmental factors, light plays a particularly important role in the successful completion of a plant's life cycle. Ultraviolet wavelengths greater than $280 \mathrm{~nm}$ are a ubiquitous component of the solar radiation, but their levels vary considerably in the biosphere both spatially and temporally (Interdonato et al., 2011). However, in recent years, ultraviolet-B (UV-B, 280 to $320 \mathrm{~nm}$ ) radiation has increased more than ever before. Exposure to such stronger UV-B radiation must have some negative impacts on plants (Sunkar, 2010). Plants cannot escape from their surroundings; therefore, to adapt to changing environmental factors and respond to a variety of stressful conditions, plants must adjust the expression of a variety of genes at the transcriptional, post-transcriptional, and posttranslational levels to enhance their tolerance at the biochemical and physiological levels (McKenzie et al., 2007). Several hundred stress-responsive genes have been identified in plants to date (Shukla et al., 2008), and microRNAs (miRNAs) have emerged as important post-transcriptional regulators in plant stress responses (Yin and Shen, 2010).

MiRNAs are a class of negative post-transcriptional regulators that are roughly 20-ntlong, noncoding RNAs and have been intensively studied (Chen, 2005; Sunkar et al., 2007). These miRNAs are produced from precursors with unique stem-loop structures (Kidner and Martienssen, 2005). They regulate various aspects of developmental programs, including auxin signaling, meristem boundary formation, and organ separation (Bartel, 2004). It has also been reported that miRNAs are involved in plant responses to biotic and abiotic stresses (Jones-Rhoades and Bartel, 2004). Some miRNAs change their level of expression in response to stress, implying that these miRNAs could play important roles in stress tolerance. For example, the expression of 10 analyzed miRNA families was significantly repressed in galled loblolly pine (Pinus taeda) stems that were infected with the fungus Cronartium quercuum $\mathrm{f}$ sp fusiforme (Lu et al., 2007). Similarly, the level of miRNA expression has been shown to be altered in plants infected by viruses (Bazzini et al., 2007). Furthermore, the expression of specific plant miRNAs has also been shown to be altered under abiotic stresses, such as cold, drought, salinity, phosphate or sulfate starvation, oxidative stress, or mechanical stress. Twenty-one miRNAs, belonging to the following 11 miRNA families, were identified in Arabidopsis and predicted to be upregulated under UV-B stress: miR156/157, miR159/319, miR160, miR165/166, miR167, miR169, miR170/171, miR172, miR393, miR398, and miR401 (Zhou et al., 2007). In Populus tremula, miR156, miR160, miR165/166, miR167, miR398, and miR168 were also reported to be upregulated by UV-B radiation (Jia et al., 2009).

Wheat (Triticum aestivum) is the most widely grown crop plant, and its yield is mainly limited by various environmental factors. Wheat miRNAs have been widely recognized as important regulatory elements, and many computational predictions of potential wheat miRNAs have already been made (Han et al., 2009). Novel miRNAs have been uncovered by deep sequencing of small RNA transcriptomes in bread wheat (Wei et al., 2009). In addition, some wheat miRNAs have been identified and proven to responsive to powdery mildew infection and heat stress (Xin et al., 2010). However, UV-B-responsive miRNAs have not been identified by either computational or experimental approaches in wheat, and the responses of miRNAs to the UV-B stress pathway are poorly understood. We carried out this experiment to unravel the complex mechanism of gene regulation under UV-B radiation in wheat. 


\section{MATERIAL AND METHODS}

\section{Plant materials and stress treatments}

Wheat genotype Suwon11 was grown in a growth chamber at a relative humidity of $75 \%$ and $26 / 20^{\circ} \mathrm{C}$ day/night temperatures. For the UV-B treatment, 10-day-old wheat seedlings were exposed to UV-B emitted from a bank of Philips TL30W/01 experimental lamps (emission peak, 310 to $315 \mathrm{~nm}$ ) covered with cellulose acetate to remove radiation below 290 $\mathrm{nm}$. The supplemental biologically effective UV-B daily dose, weighted using the generalized plant action spectrum normalized at $300 \mathrm{~nm}$, was $0.43 \mathrm{~W} / \mathrm{m}^{2}\left(22 \mathrm{~kJ} / \mathrm{m}^{2} \cdot\right.$ day $)$. Untreated wheat plants were used as controls. The leaves were harvested at $0,1,2,6,12$, or $24 \mathrm{hpt}$ (hours post treatment) of UV-B exposure.

All collected samples were immediately frozen in liquid nitrogen and stored at $-80^{\circ} \mathrm{C}$ prior to total RNA extraction. Three independent biological replications were performed for each treatment.

\section{Data sets}

Candidate miRNAs were selected from a wheat small RNA library that had been built in our laboratory. Wheat miRNAs were predicted using the method of Yao et al. (2007). First, we removed the coding RNA remnants of the cloned small RNAs using a BLASTN search against the wheat expressed sequence tag (EST) database from the NCBI; then, the small RNA sequences were used for fold-back secondary structure prediction using the mfold program (Zuker, 2003). Small RNAs whose precursor sequences possessed the qualified hairpinshaped secondary structure were selected as candidate miRNAs.

The upstream sequences (1000 bp) of the pre-miRNAs obtained from the NCBI were retrieved to identify cis-acting motifs using PlantCARE (http://bioinforma- tics.psb.ugent.be/ webtools/plantcare/). Target predictions were identified by searching the wheat EST and the NCBI nucleotide (NT) databases using psRNA targets (http://plantgrn.noble.org/psRNATarget/).

\section{Northern blot analysis of miRNA expression}

Candidate miRNAs were selected from the miRNAs database established by our laboratory. Then, we designed DNA oligonucleotides to be used as probes in northern blotting (Supplementary Material).

Total RNA $(50 \mu \mathrm{g})$ isolated from the wheat leaf tissue was run on a denaturing $15 \%$ Urea-PAGE gel, transferred to a Hybond- $\mathrm{N}^{+}$membrane, blotted, and finally probed using DNA oligonucleotides labeled with $\left[\gamma_{-32}{ }^{32} \mathrm{P}\right]$-ATP. Washed membranes were placed on Phosphor-Imager screens and subsequently scanned using a Typhoon scanner (FLA-7000 Fujifilm).

\section{Analysis of target gene expression by qRT-PCR}

All quantitative PCRs were performed in a CFX96_Real-Time system (Bio-Rad) using SYBR Green I (Invitrogen). ATP sulfurylase (APS) gene primers were designed using 
Primer 5 software as followings: sense, 5'-TAAGTAGTGCGGCGGAGGTG-3'; antisense, 5'-GACGGGCGTTGAAATGAAGTG-3' and Ta74774 primers were designed as followings: sense, 5'-GTTCACAACATGATTTAGTCAC-3'; antisense, 5'-CAAGTCCAGGTAGCTCATAT -3'. The wheat EF gene was used as a control (sense, 5'-TGGTGTCATCAAGCCTGGTATGGT-3'; antisense, 5'-ACTCATGGTGCATCTCAACGGACT-3'). The PCR conditions were as follows: $94^{\circ} \mathrm{C}$ for $1 \mathrm{~min} ; 25$ cycles of $94^{\circ} \mathrm{C}$ for $15 \mathrm{~s}, 52^{\circ} \mathrm{C}$ for $15 \mathrm{~s}$, and $72^{\circ} \mathrm{C}$ for $10 \mathrm{~min}$; and a final $72^{\circ} \mathrm{C}$ for $5 \mathrm{~min}$.

\section{RESULTS}

\section{Identification of novel UV-B-responsive miRNAs in wheat}

To identify novel miRNAs in wheat, all of the sequences of the miRNAs were mapped to selected genomes (wheat transcript assemblies from TIGR and our laboratory). A novel miRNA, named Tae-miR6000, was induced in response to UV-B radiation. The miR6000 precursor (Ta101613) contained a stable and conserved stem-loop structure (Figure 1A). The target of miR6000 was a hypothetical protein (Ta74774) in wheat (Figure 1B). To validate and analyze the expression of miR6000 in wheat treated by UV-B, we performed a northern blot analysis. The results showed that the expression of miR6000 was significantly upregulated at $1 \mathrm{hpt}$, and downregulated at the other time points (Figure 2).

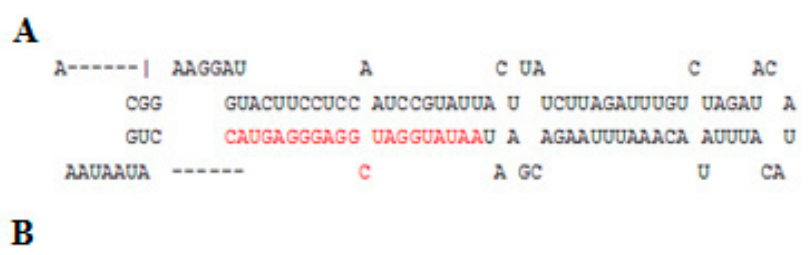

$\operatorname{miR} 6000$

$\mathrm{Ta} 7477$

5'-AAUAUGGAUCGGAGGGAGUAC-3' $\mathrm{x}|||||||\mathrm{x}||||||||||| \mathrm{x}$ 3'-UAGUUCACAACAUGaUUUAGUCACUgUGAaCUAUACCUUGCCUCCCUCAUCGUACAUAUAUGAG-5'

Figure1. Stem-loop structure of miR6000 miRNA precursor. A. miRNA sequence is in red, 5'-AAUAUGGAUCG GAGGGAGUAC-3'. B. Wheat unigenes Ta74774, the predicted targets of miR6000.

\section{RNA blotting validation of the UV-B-regulated miRNAs}

To gain insight into the possible roles of miRNAs in wheat UV-B stress regulation, we examined the expression patterns of 44 Tae-miRNAs from the northern blot analysis in miRBase Release 18 (http://www.mirbase.org/). The expression levels of six miRNAs were altered in wheat treated by UV-B at each of the six time points described above (Figure 2). Three miRNAs generally tended to be downregulated. Of these three downregulated miRNAs, miR164 and miR395 were obviously downregulated at $12 \mathrm{hpt}$, whereas the lowest expression of miR156 was at $1 \mathrm{hpt}$. In contrast, the expression levels of miR159 and miR167 peaked at $24 \mathrm{hpt}$, and miR171 was the highest expressed at $2 \mathrm{hpt}$. 


\section{Ohpt 1hpt 2hpt 6hpt $12 \mathrm{hpt} 24 \mathrm{hpt}$}

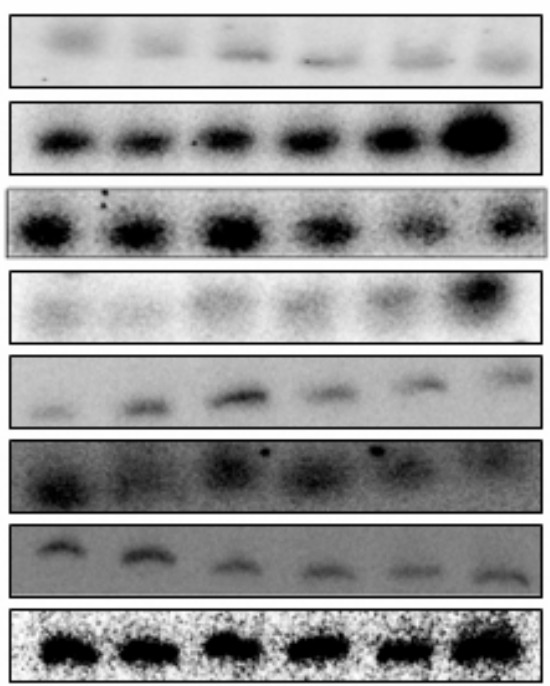

$\operatorname{miR} 156$

$\operatorname{miR} 159$

$\operatorname{miR} 164$

$\operatorname{miR} 167 \mathrm{a}$

$\operatorname{miR} 171$

$\operatorname{miR} 395$

$\operatorname{miR} 6000$

U6

Figure 2. Wheat miRNAs response to UV-B by Northern blot. Total RNA $(50 \mu \mathrm{g})$ was used for Northern blot analysis. U6 was used as a loading control.

\section{Analysis of potential targets of miRNAs}

To better understand whether the targets also responded to the UV-B, we selected targets of miR6000 and miR395 for transcript analysis. We predicted that the targets of miR6000 and miR395 were Ta74774 and APS in wheat, using psRNA targets. It was reported that APS was induced by UV-B in other plants (Jia et al., 2009), and Ta74774 as a novel miRNA target was the first to be identified under the UV-B condition. In general, miRNAs and their targets may show opposite expressions after being induced. The qRT-PCR results showed that under UV-B condition, Ta74774 was downregulated at $1 \mathrm{hpt}$, and upregulated at the other four time points. APS was upregulated at five time points, and peaked at $1 \mathrm{htp}$ (Figure 3). These results were mostly opposite to the miRNAs expression.

\section{Cis-elements in the promoters of miRNA genes in wheat}

To further explore the miRNA-mediated molecular pathway in the plant response to UV-B, we analyzed cis-elements in the promoters of pre-miRNA genes in wheat using PlantCARE. It has been proven that cis-elements analysis is helpful for understanding why miRNAs play roles under abiotic stress (Zhou et al., 2007; Jia et al., 2009). Two light-related motifs, the I-box (miR167a) and the G-box (miR156, miR159, miR167a, and miR395), were found in the miRNAs. Additionally, there were some abiotic stress-related cis-elements, such as the MYB binding site (MBS), anaerobic response element (ARE), and TC-rich motifs, in the promoters of the miRNA genes (Table 1). However, the pre-miRNA gene sequences of miR171 were not long enough to analyze. 
Table 1. Major predicted light-related and stress-responsive cis-elements in the upstream regions of UV-B-responsive miRNA genes G-box (CACGTG), I-box (GATAA), stress-responsive cis-elements: MYB binding site (MBS), anaerobic response element (ARE), TATA-box (TATAAA), CAAT-box (GGCCAATCT), and TC-rich repeats (ATTTTCTTCA).

\begin{tabular}{lccccccc}
\hline & I-box & G-box & ARE & MBS & TATA-box & CAAT-box & TC-rich \\
\hline miR159 & 2 & 1 & 3 & 2 & & 3 & 1 \\
$\operatorname{miR} 167$ & 1 & & & 1 & 1 & 1 \\
$\operatorname{miR} 156$ & & 1 & & 3 & 1 & 1 & 1 \\
$\operatorname{miR} 395$ & & 1 & & & 1 & & 1 \\
miR164 & & & & & & 1 & 1 \\
\hline
\end{tabular}

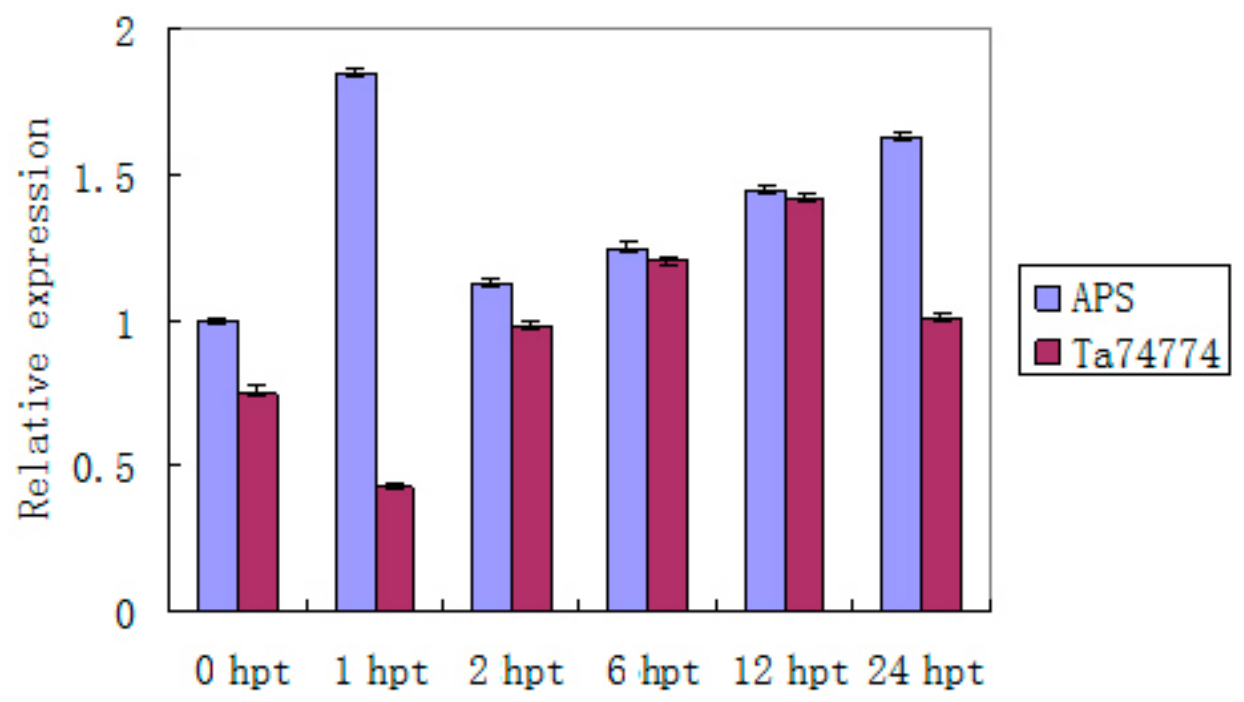

Figure 3. Analyses of transcripts of Ta74774 and APS in wheat under UV-B stress by qRT-PCR.

\section{DISCUSSION}

The whole hexaploid wheat genome is approximately 40 times larger than that of rice; therefore, wheat miRNA pathways are likely more complicated (Feuillet and Keller, 2002; Paolacci et al., 2009). Furthermore, the sequencing of the wheat genome is not currently complete. Hence, it is difficult to explore the function of miR6000 as a novel wheat miRNA. However, the miR6000 expression was highly repressed in the wheat response to UV-B stress. Its putative target Ta74774 was also induced by UV-B, and showed the opposite expression to miR6000. There may also be other unknown targets of miR6000. This result indicates that miR6000 may play an important role in UV-B abiotic stress and that its function should differ at different times based on the plant's expression of this miRNA.

The characterization of miRNAs involved in plant stress responses is an active area of research (Phillips et al., 2007). The differential expression of abiotic stress responsive miRNA genes can be considered as an adaptation of the changing environment. In Arabidopsis, miR156 and miR164 were induced by infection with the plant virus TYMV p69, and also induced 
in transgenic Arabidopsis plants expressing the viral silencing suppressor P1/HC-Pro (Kasschau et al., 2003; Chen et al., 2004). Kim et al. (2009) demonstrated that ORE1, which is an NAC transcription factor, positively regulated aging-induced cell death in Arabidopsis leaves. ORE1 expression was up- regulated concurrently with leaf aging by EIN2, but was negatively regulated by miR164 (Kim et al., 2009). That seemed to prove that miR164 has some relations with some stress factors. Furthermore, miR319c, miR389a, miR397b, and miR402 were regulated by different abiotic stress treatments to varying degrees in Arabidopsis (Sunkar and Zhu, 2004). MiR398, downregulated by oxidative stress, resulted in accumulation of its targets (CDS1 and CSD2 mRNAs) in Arabidopsis (Sunkar et al., 2006). Several miRNA levels were differentially regulated in maize seedlings exposed to submergence, where miR167, miR166, miR171, and miR396 were induced during the early phase of submergence. On the other hand, miR159, miR474, and miR528 were downregulated during the early phase but induced after long-term exposure (Zhang et al., 2008). Interestingly, the numbers of the miR169 family were upregulated in rice and Arabidopsis after salt stress (Zhao et al., 2009).

Elevated UV-B is known to negatively affect plant growth and development. UV-B radiation triggers physiological, biochemical, and molecular changes, resulting in adaptive responses in plants. The involvement of plant miRNAs in response to UV-B radiation had been convincingly demonstrated, but the role of miRNAs in the wheat response to UV-B radiation was still unknown. In our study, we found that miR167a was upregulated in wheat after UV-B treatments, which is similar to the result observed in Populus. However, most of the miRNAs studied in this research showed different expression patterns in response to UV-B radiation in different plants. Tae-miR159 was upregulated in wheat in response to UV-B radiation, but the levels of ptc-miR159 expression were lower in Populus. Similarly, miR408 was highly induced in Populus, but not changed in response to UV-B radiation in wheat and Arabidopsis. Taken together, these results suggest that each plant has its own special UV-B-regulated network.

Researching miRNA targets helps us to gain a better understanding of the mechanism of responses to abiotic stress. The expression of miR395 was increased upon sulfate starvation, showing that miRNAs can be induced by environmental factors and not only by developmental processes in Arabidopsis. The targets of miR395 were the APS (APS1, APS3, and APS4) enzymes that catalyze the first step of inorganic sulfate assimilation. The expression of miR395 depends on sulfate levels, and the APS1 expression declining with increasing miR395 corroborated the concept that this miRNA regulates sulfate metabolism (Jones-Rhoades and Bartel, 2004; Phillips et al., 2007). However, a limited number of candidate targets have been reported in wheat, to date. In this study, we predicted the targets of some wheat miRNAs using model plants. One target for miR395 was predicted to code for APS as well. It has been reported that UV-B radiation suppressed the expression of miR395 and upregulated the expression of APS1 in Populus (Jia et al., 2009). Similarly, we found that the wheat miR395 and APS may function inversely. We further inferred that the repression of miR395 may activate the sulfur assimilation pathway to form sufficient metabolites to defend cells from damage by UV-B radiation.

In our study, the analysis of cis-elements showed that miR159 contained the I-box, Gbox, and other cis-elements such as MBS and AREs (Allen et al., 2007; Reyes and Chua, 2007; Zhu et al., 2010) and TC-rich motifs, which indicates that miR159 may be involved in multiple pathways that react to UV-B stress. With regards the function of miR159, some evidence have shown that miR159 was responsive to hormone signaling and dehydration. These studies reported that miR159 regulated the abscisic acid (ABA) and gibberellic acid signaling pathways 
in the plant developmental context, and that ABA could induce miR159 expression during seed development, which negatively regulated MYB33 and MYB101. Based on these previous results, we hypothesized that wheat miR159 was induced not only to protect damage from UV-B radiation but also to adjust the physical activities of wheat plants at a late stage of UV-B stress.

In this study, families of miR156, miR159, miR164, miR167a, miR171, and miR395 were responsive to UV-B stress and showed different expression patterns. This result indicates that various miRNAs were cross-talking when the plant was under stressful conditions in order to adapt to the environmental changes. In addition, abiotic stress responses may share some common miRNAs with biotic stress responses. For example, Xin et al. (2010) reported that nine wheat miRNAs were co-regulated by both powdery mildew infection and heat stress. In summary, plant miRNAs may be involved in a complicated regulation network to respond to environmental stress.

\section{ACKNOWLEDGMENTS}

Research supported by National Key Basic Research Program of China (\#2013CB127700), the Nature Science Foundation of China (\#31071651), and the "111" Project from the Ministry of Education of China (\#B07049). We thank Professors Guiliang Tang, Yijun Qi, and Jianmin Zhou for their careful directions.

\section{REFERENCES}

Agarwal M, Hao Y, Kapoor A, Dong CH, et al. (2006). A R2R3 type MYB transcription factor is involved in the cold regulation of CBF genes and in acquired freezing tolerance. J. Biol. Chem. 281: 37636-37645.

Allen RS, Li J, Stahle MI, Dubroue A, et al. (2007). Genetic analysis reveals functional redundancy and the major target genes of the Arabidopsis miR159 family. Proc. Natl. Acad. Sci. U. S. A. 104: 16371-16376.

Bartel DP (2004). MicroRNAs: genomics, biogenesis, mechanism, and function. Cell 116: 281-297.

Bazzini AA, Hopp HE, Beachy RN and Asurmendi S (2007). Infection and coaccumulation of tobacco mosaic virus proteins alter microRNA levels, correlating with symptom and plant development. Proc. Natl. Acad. Sci. U. S. A. 104: 12157-12162.

Chen X (2005). MicroRNA biogenesis and function in plants. FEBS Lett. 579: 5923-5931.

Chen J, Li WX, Xie D, Peng JR, et al. (2004). Viral virulence protein suppresses RNA silencing-mediated defense but upregulates the role of microRNA in host gene expression. Plant Cell 16: 1302-1313.

Feuillet C and Keller B (2002). Comparative genomics in the grass family: molecular characterization of grass genome structure and evolution. Ann. Bot. 89: 3-10.

Han Y, Luan F, Zhu H, Shao Y, et al. (2009). Computational identification of microRNAs and their targets in wheat (Triticum aestivum L.). Sci. China C Life Sci. 52: 1091-1100.

Interdonato R, Rosa MC, Nieva B, Gonzalez A, et al. (2011). Effects of low UV-B doses on the accumulation of UV-B absorbing compounds and total phenolics and carbohydrate metabolism in the peel of harvested lemons. Environ. Exp. Bot. 70: 204-11.

Jia X, Ren L, Chen QJ, Li R, et al. (2009). UV-B-responsive microRNAs in Populus tremula. J. Plant. Physiol. 166: 2046-2057.

Jones-Rhoades MW and Bartel DP (2004). Computational identification of plant microRNAs and their targets, including a stress-induced miRNA. Mol. Cell 14: 787-799.

Kasschau KD, Xie Z, Allen E, Llave C, et al. (2003). P1/HC-Pro, a viral suppressor of RNA silencing, interferes with Arabidopsis development and miRNA unction. Dev. Cell 4: 205-217.

Kidner CA and Martienssen RA (2005). The developmental role of microRNA in plants. Curr. Opin. Plant Biol. 8: 38-44.

Kim JH, Woo HR, Kim J, Lim PO, et al. (2009). Trifurcate feed-forward regulation of age-dependent cell death involving miR164 in Arabidopsis. Science 323: 1053-1057.

Lu S, Sun YH, Amerson H and Chiang VL (2007). MicroRNAs in loblolly pine (Pinus taeda L.) and their association with fusiform rust gall development. Plant J. 51: 1077-1098. 
McKenzie RL, Aucamp PJ, Bais AF, Bjorn LO, et al. (2007). Changes in biologically-active ultraviolet radiation reaching the Earth's surface. Photoch. Photobil. Sci. 6: 218-31.

Paolacci AR, Tanzarella OA, Porceddu E and Ciaffi M (2009). Identification and validation of reference genes for quantitative RT-PCR normalization in wheat. BMC Mol. Biol. 10: 11.

Phillips JR, Dalmay T and Bartels D (2007). The role of small RNAs in abiotic stress. FEBS Lett. 581: 3592-3597.

Reyes JL and Chua NH (2007). ABA induction of miR159 controls transcript levels of two MYB factors during Arabidopsis seed germination. Plant J. 49: 592-606.

Shukla LI, Chinnusamy V and Sunkar R (2008). The role of microRNAs and other endogenous small RNAs in plant stress responses. Biochim. Biophys. Acta 1779: 743-748.

Sunkar R and Zhu JK (2004). Novel and stress-regulated microRNAs and other small RNAs from Arabidopsis. Plant.Cell 16: 2001-2019.

Sunkar R, Kapoor A and Zhu JK (2006). Posttranscriptional induction of two Cu/Zn superoxide dismutase genes in Arabidopsis is mediated by downregulation of miR398 and important for oxidative stress tolerance. Plant Cell 18: 2051-2065.

Sunkar R, Chinnusamy V, Zhu J and Zhu JK (2007). Small RNAs as big players in plant abiotic stress responses and nutrient deprivation. Trends Plant Sci. 12: 301-309.

Sunkar R (2010). MicroRNAs with macro-effects on plant stress responses. Semin. Cell Dev. Biol. 21: 805-811.

Wei B, Cai T, Zhang R, Li A, et al. (2009). Novel microRNAs uncovered by deep sequencing of small RNA transcriptomes in bread wheat (Triticum aestivum L.) and Brachypodium distachyon (L.) Beauv. Funct. Integr. Genomics 9: 499511.

Xin M, Wang Y, Yao Y, Xie C, et al. (2010). Diverse set of microRNAs are responsive to powdery mildew infection and heat stress in wheat (Triticum aestivum L.). BMC Plant Biol. 10: 123.

Yao Y, Guo G, Ni Z, Sunkar R, et al. (2007). Cloning and characterization of microRNAs from wheat (Triticum aestivum L.). Genome Biol. 8: R96.

Yin ZJ and Shen FF (2010). Identification and characterization of conserved microRNAs and their target genes in wheat (Triticum aestivum). Genet. Mol. Res. 9: 1186-1196.

Zhang Z, Wei L, Zou X, Tao Y, et al. (2008). Submergence-responsive microRNAs are potentially involved in the regulation of morphological and metabolic adaptations in maize root cells. Ann. Bot. 102: 509-519.

Zhao B, Ge L, Liang R, Li W, et al. (2009). Members of miR-169 family are induced by high salinity and transiently inhibit the NF-YA transcription factor. BMC Mol. Biol. 10: 29.

Zhou X, Wang G and Zhang W (2007). UV-B responsive microRNA genes in Arabidopsis thaliana. Mol. Syst. Biol. 3: 103.

Zhu YY, Zeng HQ, Dong CX, Yin XM, et al. (2010). MicroRNA expression profiles associated with phosphorus deficiency in white lupin (Lupinus albus. L.). Plant Sci. 178: 23-9.

Zuker M (2003). Mfold web server for nucleic acid folding and hybridization prediction. Nucleic Acids Res. 31: $3406-$ 3415. 